\title{
Association of hormone replacement therapy with mortality in colorectal cancer survivor: a systematic review and meta- analysis
}

Yeu-Chai Jang ${ }^{1 \dagger}$, Hsi-Lan Huang ${ }^{2,3+}$ and Chi Yan Leung ${ }^{2,3^{*}}$

\begin{abstract}
Background: Hormone replacement therapy (HRT) use has shown to be associated with a reduced risk of colorectal cancer, however, its impact on survival among women with colorectal cancer remains uncertain. This meta-analysis aimed to systematically assess the survival benefit of HRT use in patients with colorectal cancer.

Methods: PRISMA guidelines for the reporting of meta-analyses were followed. We systematically searched PubMed, Embase, Cochrane library, Scopus, and PsycINFO from inception to 12 January 2019, with no language restrictions, for randomized controlled trials and cohort studies reporting the association between hormone replacement therapy and risk of colorectal cancer mortality or all-cause mortality in colorectal cancer survivors. We used the Newcastle-Ottawa Scale to assess the risk of bias of the included studies. We summarized the association as hazard ratio $(\mathrm{HR} ; 95 \% \mathrm{Cl})$ using random-effects meta-analysis. The study protocol was registered in PROSPERO (CRD42017071914).

Results: Of 1648 articles identified, five cohorts including 10,013 colorectal cancer survivors were included in this meta-analysis. Compared with women with no prior use of HRT, those reporting current use of HRT had lower risks of colorectal cancer-specific mortality $\left(\mathrm{HR}, 0.71[95 \% \mathrm{Cl}, 0.62-0.80], P^{2}=0 \%\right)$ and overall mortality $(\mathrm{HR}, 0.74[95 \% \mathrm{Cl}$, $\left.0.67-0.81], P^{2}=0 \%\right)$. Low between-study variance was also suggested by the narrow prediction interval for colorectal cancer-specific mortality $(0.58-0.86)$ and overall mortality $(0.63-0.87)$, which indicated that a future study will show survival benefits in women with current HRT use compared with those with no HRT exposure. Inverse associations with colorectal cancer-specific (HR, $\left.1.02[95 \% \mathrm{Cl}, 0.82-1.28], P^{2}=0 \%\right)$ and overall mortality $(\mathrm{HR}, 1.07$ [95\% Cl, 0.901.27], $P^{2}=0 \%$ ) were not observed for former users of HRT. Sensitivity analyses revealed no differences in the risk estimates between two groups.
\end{abstract}

Conclusions: The findings suggest that the current use of HRT is associated with lower risks of colorectal cancerspecific and overall mortality in patients with colorectal cancer. Further investigations to elucidate the underlying mechanism are warranted.

Keywords: Hormone replacement therapy, Colorectal cancer survivor, Survival

\footnotetext{
* Correspondence: chiyan0509@m.u-tokyo.ac.jp

${ }^{\dagger}$ Yeu-Chai Jang and Hsi-Lan Huang contributed equally to this work.

${ }^{2}$ Department of Global Health Policy, Graduate School of Medicine, The

University of Tokyo, 7-3-1 Hongo, Bunkyo-ku, Tokyo 113-0033, Japan

${ }^{3}$ Division of Cancer Statistics Integration, Center for Cancer Control and

Information Services, National Cancer Center, Tokyo, Japan

Full list of author information is available at the end of the article
}

(c) The Author(s). 2019 Open Access This article is distributed under the terms of the Creative Commons Attribution 4.0 International License (http://creativecommons.org/licenses/by/4.0/), which permits unrestricted use, distribution, and reproduction in any medium, provided you give appropriate credit to the original author(s) and the source, provide a link to the Creative Commons license, and indicate if changes were made. The Creative Commons Public Domain Dedication waiver (http://creativecommons.org/publicdomain/zero/1.0/) applies to the data made available in this article, unless otherwise stated. 


\section{Background}

Colorectal cancer is the third most common cause of cancer mortality in women, with 0.8 million new cases in 2018 worldwide [1]. The advances in treatment of colorectal cancer has translated to a marked improvement in survivorship in the past decades [1]. From 1995 to 2014 , the 5 -year survival was increased by $5-10 \%$ in various countries [2]. Given the aging population and advances in treatment in the past decades, the 5-year colorectal cancer prevalence in women were estimated to be over 2 million in 2018 [3]. Despite these encouraging figures, the cumulative impact of cancer and ongoing chronic physical and emotional symptoms reduce quality of life and overall survival [4].

Identification of factors associated with better prognosis among colorectal cancer survivors has important implications to inform provision of survivorship care. Extensive evidence from observational studies and clinical trials suggests that hormone replacement therapy (HRT) might have protective effect against colorectal cancer incidence. A meta-analysis of four randomized control trials and 16 observational studies showed a $26 \%$ reduction in colorectal cancer risk associated with any use of combined estrogen and progestin [5]. Similar association was observed for estrogen-only HRT despite considerable between-study heterogeneity [5]. However, previous evidence only focused on the association between HRT and colorectal cancer risk [5]. The role of HRT use on risk of mortality in patients diagnosed with colorectal cancer remains uncertain. A comprehensive evaluation of the impact of hormone therapy use among cancer survivors is urgently needed to inform physicians and patients for treatment decision making.

In this systematic review and meta-analysis, we aimed to summarize the available evidence and to quantify the association of HRT with colorectal-specific and all-cause mortality in women with diagnosis of colorectal cancer.

\section{Methods}

Search strategy and selection criteria

The review protocol was registered in PROSPERO (CRD42017071914). In this study, we followed Preferred Reporting Items for Systematic Reviews and MetaAnalyses (PRISMA) guidelines for the reporting of metaanalyses (See Additional file 1: Table S1) [6]. We systematically searched PubMed, Embase, Cochrane library, Scopus, and PsycINFO from inception to 12 January 2019, with no language restrictions, reporting the association between hormone replacement therapy and risk of colorectal cancer mortality or all-cause mortality in colorectal cancer survivors. Articles were searched using keywords and Mesh terms relating to hormone replacement therapy; and colorectal, colon, or rectal cancer survivor. In addition, we searched reference lists of identified articles (Details of search strategies are described in Additional file 1: Tables S2, S3, S4, S5 and S6).

We did this systematic review and meta-analysis according to prespecified methodological criteria: (1) Published original articles of randomized controlled trials or cohort studies with a minimum sample sizes larger than 50 participants; (2) Studies that enrolled colorectal cancer survivors aged 40 to 75 years, or studies that evaluated peri- or post-menopausal women; and (3) The exposure of interest was HRT. In the meta-analysis, study must either provide hazard ratio (HR), relative risk (RR), or odds ratio (OR) with $95 \%$ confidence intervals (CIs); or provide sufficient data that would allow the risk estimate to be calculated. The primary outcome was colorectal cancer-specific mortality. Secondary outcome was all-cause mortality in survivor with colorectal cancer. We excluded case-control studies, reviews, editorials, letters, and animal studies. For publications assessing duplicate population, only studies with larger sample size were included. For non-English articles, we consulted native speakers for translation. Two reviewers (YCJ and HLH) independently screened the title and abstract of potentially eligible articles for inclusion. Disagreement on eligibility was resolved by discussion between the reviewers.

\section{Data extraction and quality assessment}

Two independent reviewers (YCJ and HLH) extracted the data from identified articles, and a third reviewer (CYL) crosschecked the abstracted data for accuracy. We extracted data using a standardized observation form, items of the form included name of first author, publication year, age, study design, study period, country; number of participants; cancer stage and grade at diagnosis; HRT type and recency; mortality (colorectal cancer and all-cause); and follow-up time. We extracted the most finely adjusted risk estimates from the included studies. The Newcastle-Ottawa scale (NOS) proposed by Well and colleagues was used to assess the methodological quality for cohort studies [7].

\section{Statistical analysis}

In the analysis, random-effects meta-analysis was used to pool the association between hormone replacement therapy and risk of colorectal cancer mortality and the risk of all-cause mortality in patients with colorectal cancer diagnosed. We assessed methodological and clinical heterogeneity by $I^{2}$ statistic, which quantifies the percentage of total variation across study results that could be explained by between-study heterogeneity rather than chance [8]. In this study, the cut-off value of low, moderate, and high heterogeneity were defined as an $I^{2}$ statistic of 25,50 , and $75 \%$, respectively [9]. As a separate analysis, the prediction interval, which describes 
the heterogeneity in a random-effects meta-analysis, was calculated to inform the variability of future treatment effects in $95 \%$ of all populations [10]. Potential publication bias and small-study effects were explored by visual inspection of funnel plots. We did not perform further statistical analysis for funnel plot asymmetric because the statistical power is limited when number of studies is fewer than 10 [11]. Also, we assessed all the articles for potential information bias. Each article was evaluated for its approach of assessing HRT exposure, which can be categorized into two groups: (1) questionnaire assessment and (2) objective documents, such as drug register or pharmacy database. To further examine the potential impact of information bias on HR estimates, we performed meta-regression where we regressed log-transformed HRs of each study by HRT exposure assessment method (objective documents or questionnaire). To assess the robustness of primary analysis, sensitivity analysis was conducted by omitting one study at a time [12]. In additional sensitivity analysis, a fixed-effect model was used to combine the data for the outcome. In this study, unless $p<0.0001$, exact $p$ values are provided. We used STATA version 15.1 (College Station, TX, USA) to analyse data.

\section{Results}

\section{Literature search}

Figure 1 presents the literature search details and process. Briefly, we identified 1648 articles after initial literature searching and exclusion of the duplicated articles. We further rejected 1591 articles after reviewing titles and abstracts, because they were irrelevant to the scope of our analysis. For the 26 articles underwent fulltext review, 17 were excluded because nine of them evaluated patients other than colorectal cancer survivors and eight reported results other than colorectal cancerspecific mortality or all-cause mortality. Two review articles, one case-control study, and one study reporting duplicate cohort were also excluded. Therefore, five unique studies met eligibility criteria. We did not identify additional article after reviewing the reference lists

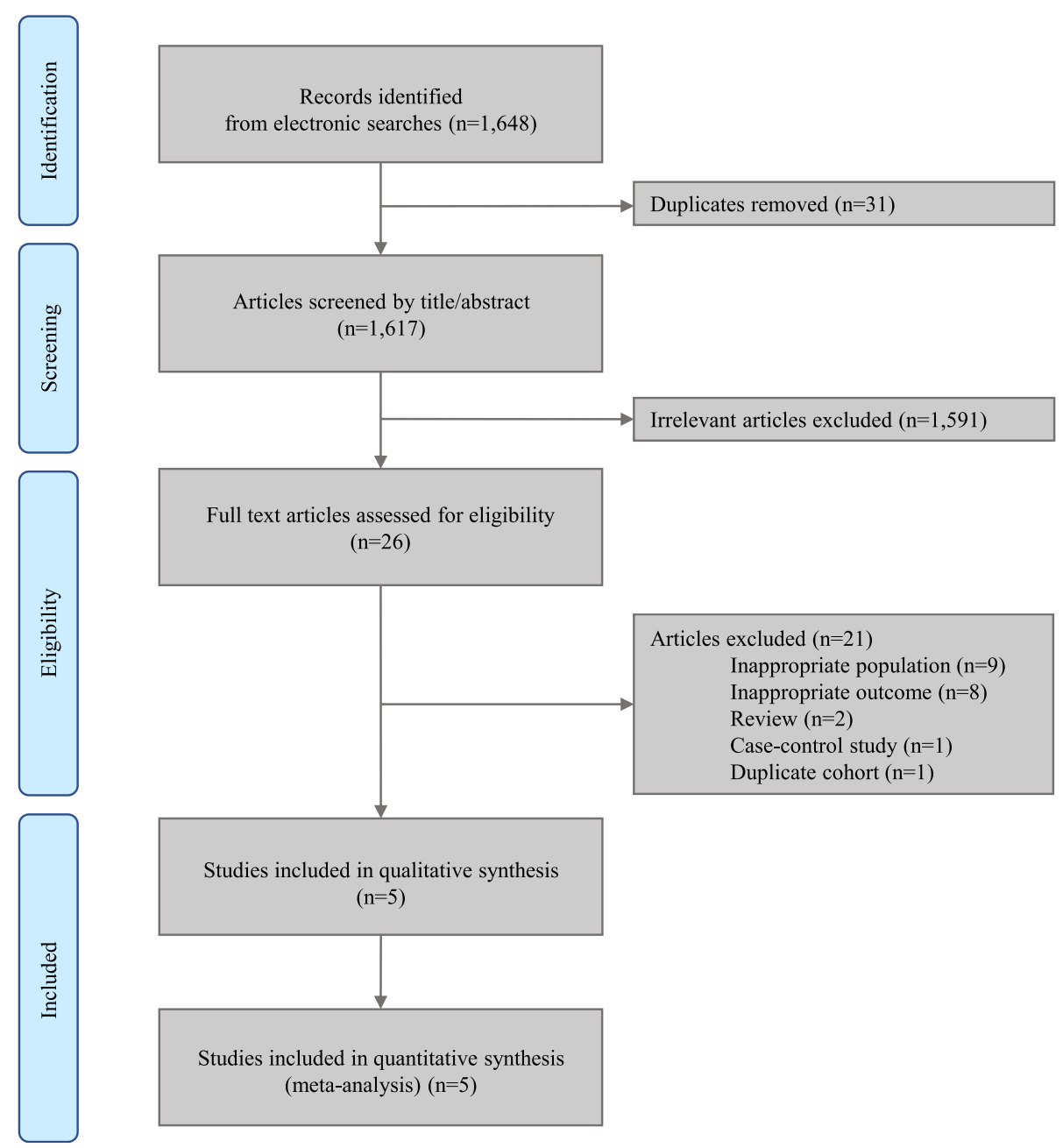

Fig. 1 Study selection. Note. n: number 
of eligible articles. Details on excluded studies are listed in the Additional file 1: Table S7.

\section{Characteristics of included studies}

Overall, four prospective cohort studies [13-16] and one retrospective cohort study [17] were included in our meta-analysis, including relevant available data on current HRT user [13-17] and former HRT user [13, 14]. Table 1 presents the baseline characteristics of the included studies. The number of colorectal cancer survivors included in each study ranged from 699 to 5626 . In total, we included 10,013 women for analysis. The studies were published from 1999 to 2018 and included patients from the United states [13-16] and Sweden [17]. Table S8 (See Additional file 1) shows the study quality assessed by NewcastleOttawa scale. The included studies are generally of low risk of bias, with Newcastle-Ottawa scale scores between seven and nine.

\section{Meta-analysis}

The meta-analysis results of pooling the five cohorts with 10,013 colorectal cancer survivors showed that the current use of HRT was associated with a significantly reduced risk of colorectal cancer mortality (HR, 0.71 [95\% CI, $0.62-0.80], I^{2}=0 \%$ ) and all-cause mortality (HR, 0.74 [95\% CI, 0.67-0.81], $I^{2}=0 \%$ ) in survivors with colorectal cancer (Fig. 2). Low between-study variance is also reflected by the narrow prediction interval (colorectal cancer mortality, 0.58-0.86; and all-cause mortality, 0.63-0.87) (Additional file 1: Figures S1 and S2). The results of prediction interval indicated that, in a future study, colorectal cancer survivors with hormone replacement therapy are likely to have a reduced mortality risk compared with non-hormone users. On the other hand, among colorectal cancer survivors, former use of HRT was not significantly associated with reduced colorectal cancer-specific mortality (HR, 1.02 [95\% CI, 0.82-1.28], $I^{2}=0 \%$ ) and all-cause mortality (HR, 1.07 [95\% CI, 0.901.27], $I^{2}=0 \%$ ) (Fig. 2).

\section{Publication bias and information bias}

We further assessed publication bias for studies evaluating the association between current use of HRT and mortality risks. Funnel plot asymmetry, which suggests the presence of publication bias and small-study effects, was not evident for included studies (Additional file 1: Figure S3 and S4). However, we did not perform statistical test to assess publication bias because limited number of eligible studies hampered the test power.

We examined the included studies for potential information bias. Among current users, the result showed no significant difference in pooled HRs of CRC-specific mortality for studies where HRT exposure was assessed by either questionnaire (HR 0.69 [95\% CI, 0.57-0.82]) or objective document (HR 0.72 [95CI\%, 0.61-0.85]) ( $p$ value for the difference $=0.6786$ ). Similarly, no significant difference was observed between questionnaire assessment (HR 0.76 [95CI\%, 0.67-0.87]) and objective document (HR 0.71 [95CI\%, 0.62-0.82]) for all-cause mortality $(p=0.486)$.

\section{Sensitivity analysis}

To assess the impact of individual studies on pooled estimates, sensitivity analysis was performed by omitting one study at a time. Figure 3 presents the pooled estimates after each study was omitted. Overall, no single study was identified to be substantially influential on the pooled estimates. For the current HRT users, omitting the most influential study by Ji et al. resulted in pooled HRs of $0.67(0.57-0.80)$ for colorectal cancer mortality and $0.76(0.67-0.87)$ for all-cause mortality, which were close to the primary analysis of $0.71(0.62-0.80)$ for colorectal cancer mortality and $0.74(0.67-0.81)$ for allcause mortality. In a second sensitivity analysis, we reran the analysis with a fixed-effect meta-analysis. The effect estimates on colorectal cancer and all-cause mortality for current and former HRT users were unchanged (Additional file 1: Figure S5 and S6).

\section{Discussion}

In this study, we performed a systematic review and meta-analysis, synthesising non-overlapping data from 10,013 colorectal cancer survivors, to quantify the association between HRT use and risk of mortality. The study findings show that the current use of HRT was associated with a significant reduction in the risk of colorectal cancer-specific mortality and all-cause mortality in women with colorectal cancer. In contrast, the former use of HRT was not associated with lower all-cause and colorectal cancer mortality in colorectal cancer survivors. Findings for the prediction interval were consistent with the main estimates. In sensitivity analysis, these results were not significantly affected in influence analysis. No differences were noted between two groups in the risk estimates using the fixed-effect meta-analysis.

Extensive evidence from clinical trials and observational studies have shown the use of HRT to be associated with a reduction in colorectal cancer risk in women [5]. In addition to the well-documented chemoprevention effects, our results also suggested a role of HRT on disease-specific and all-cause mortality among colorectal cancer survivors. There are potential mechanisms underlying the association between HRT and colorectal cancer and all-cause mortality in colorectal cancer survivors. As colorectal cancer may be a hormone-dependent cancer, tumor progression is inversely associated with expression of Estrogen Receptor Beta (ERß) [18, 19]. Konstantinopoulos and colleagues reported a significantly lower 


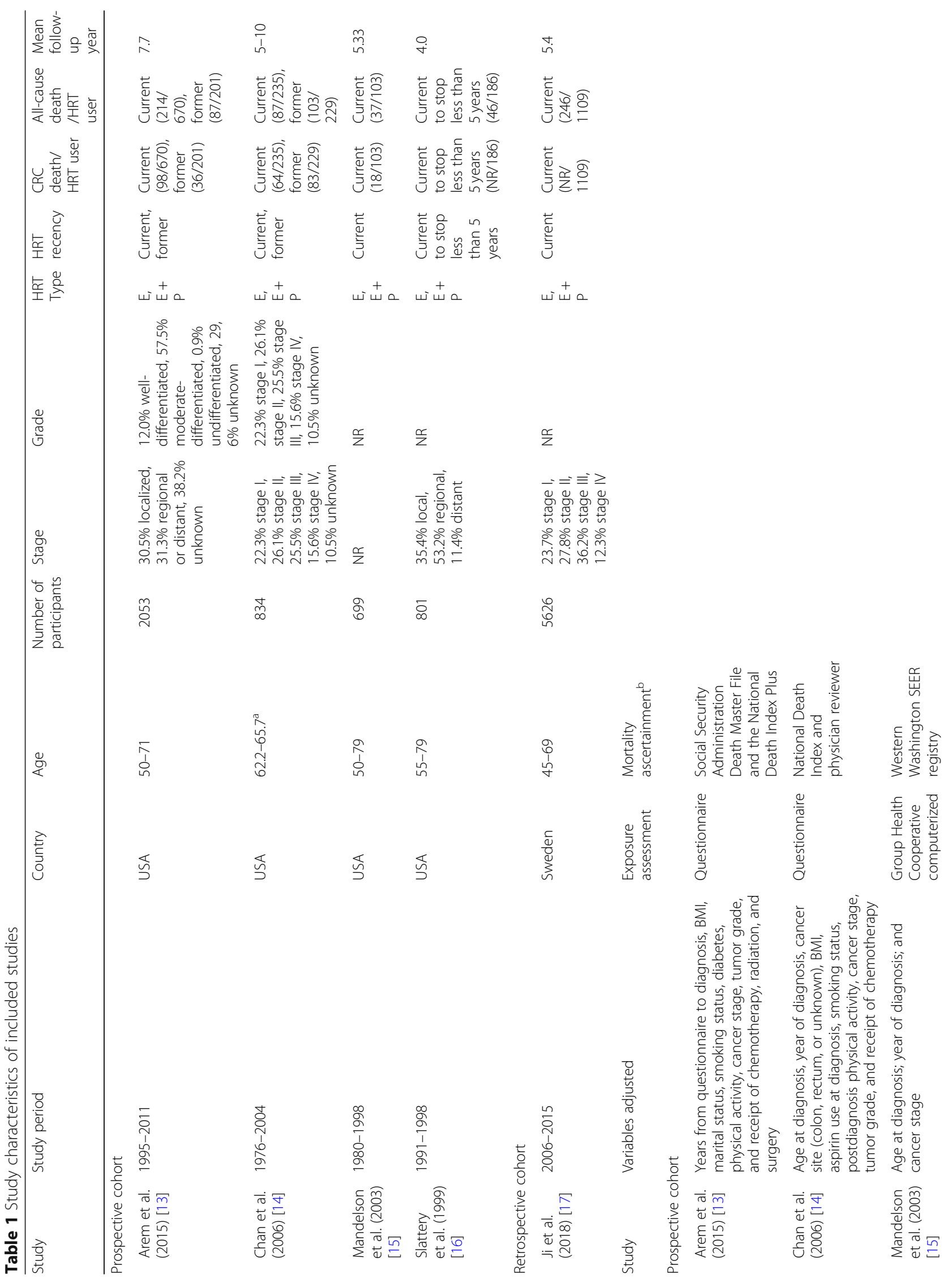




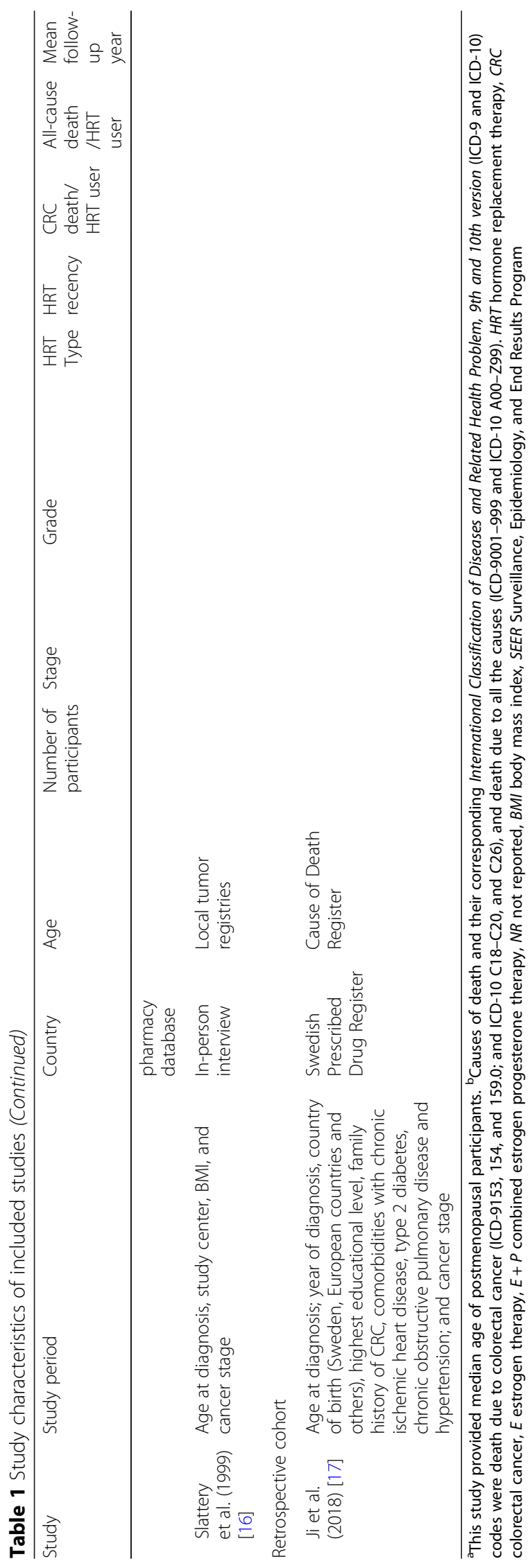



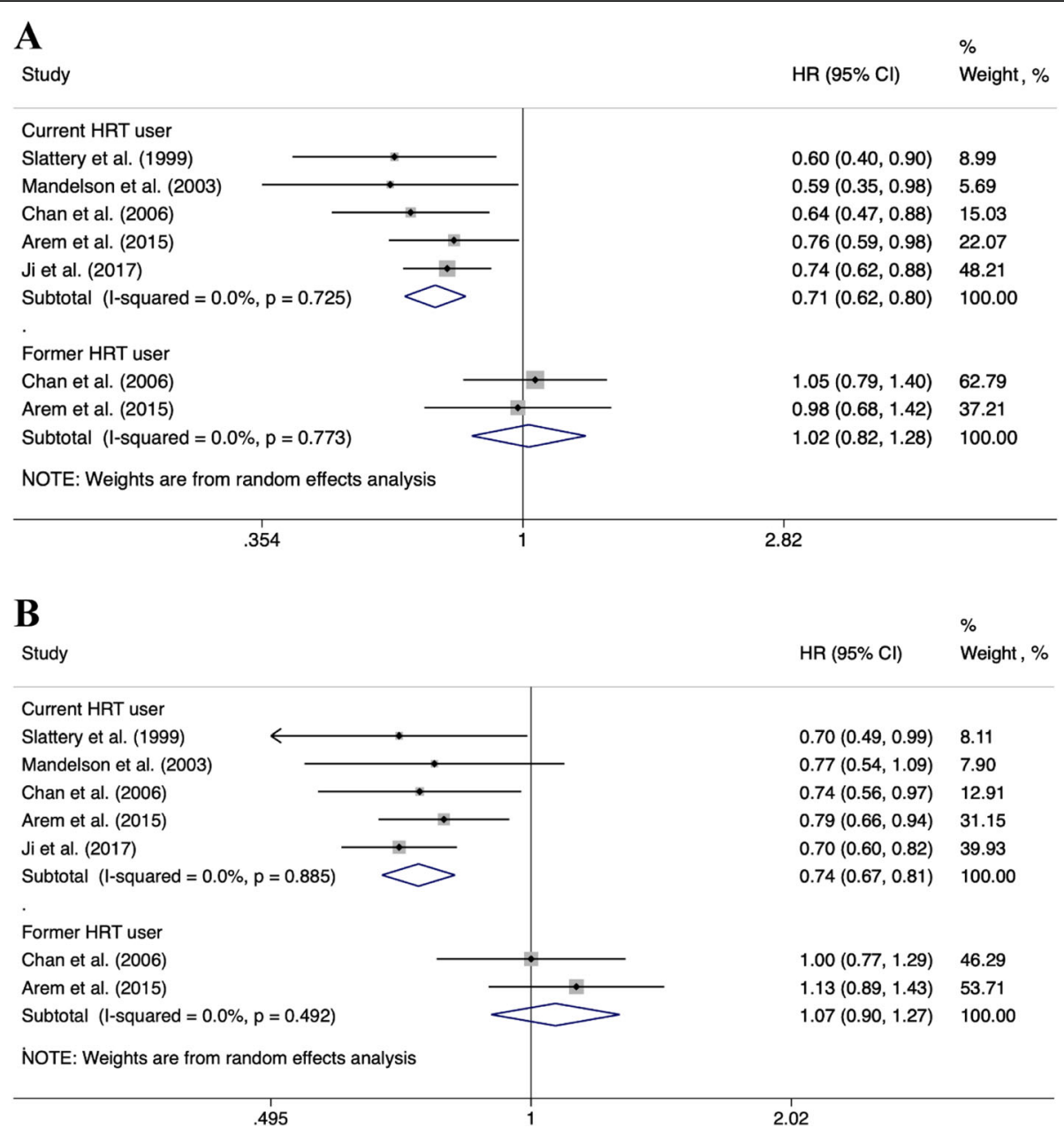

Fig. 2 Summary of pooled risk estimates. The association between HRT use in women with colorectal cancer and (a) colorectal cancers and (b) all-cause mortality. Note. HR: hazard ratio, Cl: confidence intervals, HRT: hormone replacement therapy

ERß expression in colon cancer cells compared with normal colon epithelium [20]. The reduction of ERß expression in colorectal cancer might be associated with loss of differentiation [20] and advanced cancer stages [21]. Furthermore, potential of colorectal cancer progression was reportedly repressed by ERß expression $[19,22]$. Estrogen has been demonstrated to increase ERß expression [19]. The downstream genomic protective effects of estrogen result in gene transcription related to angiogenesis and cellular adhesion. In addition, ERß has been reported to induce apoptosis via different mechanisms, including increased p53 signalling in LoVo colon cancer cells and increased DNA fragmentation in COLO205 colon cancer cells [19]. In HT29 and SW480 colon cancer cells, ERß reduces cell proliferation via modulation of G1-phase cell cycle genes [23]. Nongenomic effects of estrogen interaction with ERß include activation of various intracellular signaling pathways [24]. Estrogenic regulation of c-Myc and cyclin D1 expression contributes to the inhibition of cell cycle progression [24]. Furthermore, selective activation of ERß has an anti-carcinogenic effect on tumor microenvironment via the downregulation of inflammatory signaling (interleukin-6) [19, 22].

To date, evidence is limited to determine whether the survival benefit of HRT among colorectal cancer survivors varies by dosage, duration, or timing of initiation. In our meta-analysis, among women diagnosed with colorectal cancer, the current HRT users had a reduced mortality risk compared with women with no prior hormone exposure, whereas risk was not changed for former users, suggesting that the association between HRT use and survivorship may be complicated and depend on the timing of hormone use. While evidence suggests that the current use of HRT has a survival benefit for colorectal cancer survivors, further rigorous assessment is needed to determine whether its possible adverse events such as venous thromboembolism outweighs the benefits. Because colorectal cancer is the third most common cancer in women [1], affecting 1 in 


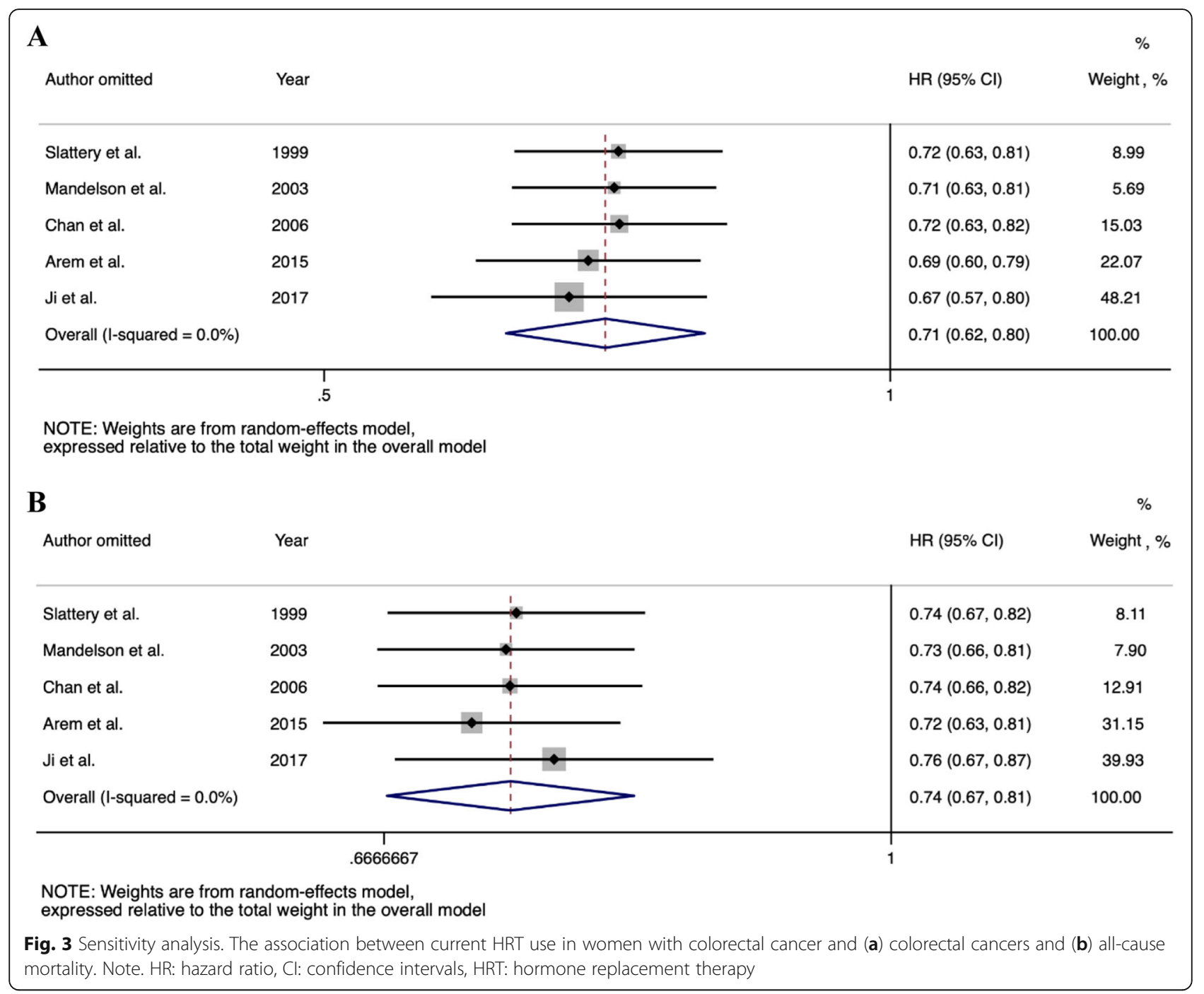

41 women over their lifetime period [25], our findings suggested that the potential favourable effects of HRT on mortality among colorectal cancer survivors justify further investigations.

\section{Strength}

To our knowledge, this is the first meta-analysis to assess the association between HRT and the risk of colorectal cancer-specific and all-cause mortality in colorectal cancer survivors. Prior reviews focused on the association between risk of colorectal cancer incidence and hormone therapy; however, our analysis provided a clinically relevant insight to the impact of HRT use on survival of patients with colorectal cancer. Another important strength of this study is that we provide predictive interval to express between-study heterogeneity in a randomeffects meta-analysis. We did not observe significant between-study variance, which was reflected by the narrow predictive interval $(0.58-0.86)$ and $(0.63-0.87)$ for colorectal cancer-specific and all-cause mortality among current HRT users, respectively. Accordingly, it is likely that a future study will show a reduced mortality risk in colorectal cancer survivors with hormone replacement therapy compared with non-hormone users.

\section{Limitation}

There are several limitations that merit further discussion. First, owing to the study-level nature of the data and small numbers of studies, subgroup analyses and the assessment of publication bias were limited. Second, although we extensively searched for the best available evidence, studies identified in this analysis were from the United States and Sweden, which has limited the generalizability of the findings. However, we have provided prediction interval to provide the potential ranges of future studies. Third, meta-analyses of observational studies are susceptible to information bias. However, four of the five studies included were prospective design, 
which could minimize information bias. Also, in our meta-regression, no significant difference was observed between questionnaire assessment group or objective document group. Fourth, the mean follow-up period of included studies were relatively short, ranging from 4.0 to 7.7 years, which may be inadequate to reflect optimal long-term outcomes. Lastly, the ability to stratify the analyses by dose, duration, and types of hormone used was limited by the paucity of data, therefore, the pooled estimates represent a combined effect of estrogen and estrogen plus progesterone. Future research is needed to understand how these factors might influence survival of perimenopausal and postmenopausal women with a history of colorectal cancer.

\section{Conclusions}

In this systematic review and meta-analysis, the current use of hormone replacement therapy was associated with a lower risk of colorectal cancer-specific mortality and all-cause mortality in colorectal cancer survivors. Further investigation is needed on the underlying mechanism to facilitate a personalized healthcare to inform cancer survivors.

\section{Supplementary information}

Supplementary information accompanies this paper at https://doi.org/10. 1186/s12885-019-6428-0.

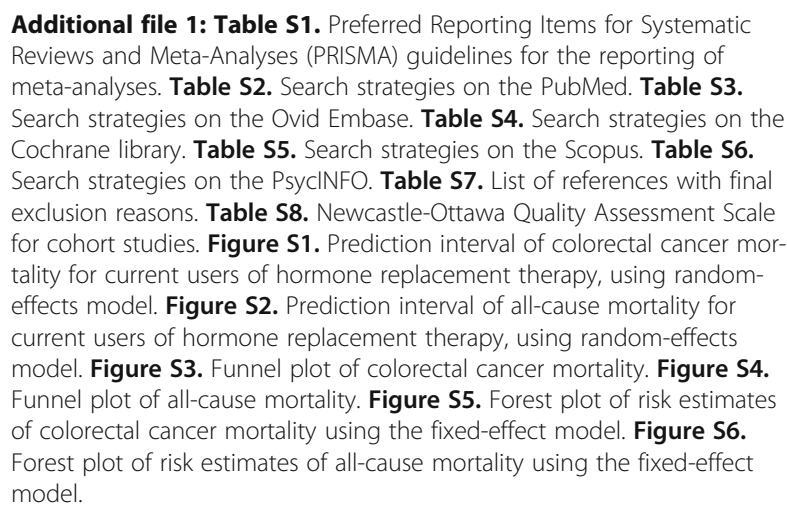

Additional file 1: Table S1. Preferred Reporting Items for Systematic Reviews and Meta-Analyses (PRISMA) guidelines for the reporting of meta-analyses. Table S2. Search strategies on the PubMed. Table S3. Search strategies on the Ovid Embase. Table S4. Search strategies on the Cochrane library. Table S5. Search strategies on the Scopus. Table S6. Search strategies on the PsycINFO. Table S7. List of references with final exclusion reasons. Table S8. Newcastle-Ottawa Quality Assessment Scale for cohort studies. Figure S1. Prediction interval of colorectal cancer mortality for current users of hormone replacement therapy, using randomeffects model. Figure S2. Prediction interval of all-cause mortality for current users of hormone replacement therapy, using random-effects model. Figure S3. Funnel plot of colorectal cancer mortality. Figure S4. Funnel plot of all-cause mortality. Figure $\mathbf{S 5}$. Forest plot of risk estimates of colorectal cancer mortality using the fixed-effect model. Figure S6. Forest plot of risk estimates of all-cause mortality using the fixed-effect model.

\section{Abbreviations}

Cl: Confidence intervals; ERß: Estrogen Receptor Beta; HR: Hazard ratio; HRT: Hormone replacement therapy; NOS: Newcastle-Ottawa scale; OR: Odds ratio; RR: Relative risk

\section{Acknowledgements}

The views expressed in this paper are solely those of the authors. The authors would like to thank Dr. LY Leung and Dr. RSL Lo, Accident and Emergency Medicine Academic Unit, Chinese University of Hong Kong for their advice on the manuscript.

\section{Authors' contributions}

YCJ, HLH, and CYL conceived and designed the study and protocol registration. YCJ and $\mathrm{HLH}$ contributed equally to this work. CYL refined the protocol registration. YCJ and HLH performed the literature search, data extraction, quality assessment. $\mathrm{HLH}$, and CYL statistical analysis, and interpretation of data. YCJ, HLH, and CYL wrote and revised the manuscript critically for important intellectual content. YCJ, HLH, and CYL had full access to all the data in the study and had final responsibility for the decision to submit for publication. All authors approved the final version before submission.

\section{Authors' information}

Dr. Yeu-Chai Jang and Dr. Hsi-Lan Huang contributed equally to this work. The Taipei Municipal Wanfang Hospital, Taiwan

Dr. Yeu-Chai Jang, MD.

Department of Global Health Policy, Graduate School of Medicine, The

University of Tokyo, Japan / Division of Cancer Statistics Integration, Center for Cancer Control and Information.

Services, National Cancer Center, Japan.

Dr. Hsi-Lan Huang, MD, MHS \& Dr. Chi Yan Leung, MD, MHS

Funding

There was no funding source for this study.

Availability of data and materials

All data generated or analysed during this study are included in this published article and its supplementary information files.

Ethics approval and consent to participate

Not applicable.

\section{Consent for publication}

Not applicable.

\section{Competing interests}

All authors declare that they have no competing interests.

\section{Author details}

${ }^{1}$ The Taipei Municipal Wanfang Hospital, Taipei, Taiwan. ${ }^{2}$ Department of Global Health Policy, Graduate School of Medicine, The University of Tokyo, 7-3-1 Hongo, Bunkyo-ku, Tokyo 113-0033, Japan. ${ }^{3}$ Division of Cancer Statistics Integration, Center for Cancer Control and Information Services, National Cancer Center, Tokyo, Japan.

Received: 4 April 2019 Accepted: 3 December 2019

Published online: 09 December 2019

\section{References}

1. Bray F, Ferlay J, Soerjomataram I, Siegel RL, Torre LA, Jemal A. Global cancer statistics 2018: GLOBOCAN estimates of incidence and mortality worldwide for 36 cancers in 185 countries. CA Cancer J Clin. 2018;68:394-424.

2. Allemani C, Matsuda T, Di Carlo V, Harewood R, Matz M, Nikšić M, et al. Global surveillance of trends in cancer survival 2000-14 (CONCORD-3): analysis of individual records for 37513025 patients diagnosed with one of 18 cancers from 322 population-based registries in 71 countries. Lancet. 2018;391:1023-75

3. Ferlay J, Ervik M, Lam F, Colombet M, Mery L, Piñeros M, Znaor A, Soerjomataram I, Bray F. Global Cancer observatory: Cancer today. Lyon: International Agency for Research on Cancer; 2018. Available from: https://gco.iarc.fr/today, Accessed 15 Mar 2019

4. Alfano CM, Leach CR, Smith TG, Miller KD, Alcaraz KI, Cannady RS, et al. Equitably improving outcomes for cancer survivors and supporting caregivers: a blueprint for care delivery, research, education, and policy. CA Cancer J Clin. 2019:69:35-49.

5. Lin KJ, Cheung WY, Lai JY, Giovannucci EL. The effect of estrogen vs. combined estrogen-progestogen therapy on the risk of colorectal cancer. Int J Cancer. 2012;130:419-30.

6. Moher D, Liberati A, Tetzlaff J, Altman DG, Prisma Group. Preferred reporting items for systematic reviews and meta-analyses: the PRISMA statement. BMJ. 2009:339:b2535.

7. Wells G, Shea B, O'Connell D. Proceedings of the Third Symposium on Systematic Reviews. Beyond the Basics: Improving Quality and Impact. Oxford: The Newcastle-Ottawa Scale (NOS) for assessing the quality of nonrandomised studies in meta-analysis; 2000.

8. Higgins JP, Thompson SG. Quantifying heterogeneity in a meta-analysis. Stat Med. 2002;21:1539-58. 
9. Higgins J, Green S. Cochrane handbook for systematic reviews of interventions version 5.1.0. London: Cochrane Collaboration; 2011.

10. IntHout J, loannidis JP, Rovers MM, Goeman JJ. Plea for routinely presenting prediction intervals in meta-analysis. BMJ Open. 2016;6:e010247.

11. Sterne JA, Sutton AJ, loannidis JP, Terrin N, Jones DR, Lau J, et al. Recommendations for examining and interpreting funnel plot asymmetry in meta-analyses of randomised controlled trials. BMJ. 2011;343:d4002.

12. Patsopoulos NA, Evangelou E, Ioannidis JP. Sensitivity of between-study heterogeneity in meta-analysis: proposed metrics and empirical evaluation. Int J Epidemiol. 2008;37:1148-57.

13. Arem H, Park Y, Felix AS, Zervoudakis A, Brinton LA, Matthews CE, et al. Reproductive and hormonal factors and mortality among women with colorectal cancer in the NIH-AARP diet and health study. Br J Cancer. 2015;113:562-8.

14. Chan JA, Meyerhardt JA, Chan AT, Giovannucci EL, Colditz GA, Fuchs CS. Hormone replacement therapy and survival after colorectal cancer diagnosis. J Clin Oncol. 2006;24:5680-6.

15. Mandelson MT, Miglioretti D, Newcomb PA, Harrison R, Potter JD. Hormone replacement therapy in relation to survival in women diagnosed with colon cancer. Cancer Causes Control. 2003;14:979-84.

16. Slattery ML, Anderson K, Samowitz W, Edwards SL, Curtin K, Caan B, et al. Hormone replacement therapy and improved survival among postmenopausal women diagnosed with colon cancer (USA). Cancer Causes Control. 1999:10:467-73.

17. Ji J, Sundquist J, Sundquist K. Use of hormone replacement therapy improves the prognosis in patients with colorectal cancer: a populationbased study in Sweden. Int J Cancer. 2018;142:2003-10.

18. Kennelly R, Kavanagh DO, Hogan AM, Winter DC. Oestrogen and the colon: potential mechanisms for cancer prevention. Lancet Oncol. 2008;9:385-91.

19. Williams C, DiLeo A, Niv Y, Gustafsson JÅ. Estrogen receptor beta as target for colorectal cancer prevention. Cancer Lett. 2016;372:48-56.

20. Konstantinopoulos PA, Kominea A, Vandoros G, Sykiotis GP, Andricopoulos $P$, Varakis I, et al. Oestrogen receptor beta (ER $\beta$ ) is abundantly expressed in normal colonic mucosa, but declines in colon adenocarcinoma paralleling the tumour's dedifferentiation. Eur J Cancer. 2003:39:1251-8.

21. Rudolph A, Toth C, Hoffmeister M, Roth W, Herpel E, Jansen L, et al. Expression of oestrogen receptor $\beta$ and prognosis of colorectal cancer. Br J Cancer. 2012;107:831-9.

22. Caiazza F, Ryan EJ, Doherty G, Winter DC, Sheahan K. Estrogen receptors and their implications in colorectal carcinogenesis. Front Oncol. 2015;5:19.

23. Hartman J, Edvardsson K, Lindberg K, Zhao C, Williams C, Ström A, et al. Tumor repressive functions of estrogen receptor $\beta$ in SW480 colon cancer cells. Cancer Res. 2009:69:6100-6.

24. Barzi A, Lenz AM, Labonte MJ, Lenz HJ. Molecular pathways: estrogen pathway in colorectal cancer. Clin Cancer Res. 2013;19:5842-8.

25. Fitzmaurice C, Akinyemiju TF, Al Lami FH, Alam T, Alizadeh-Navaei R, Allen C, et al. Global, regional, and national cancer incidence, mortality, years of life lost, years lived with disability, and disability-adjusted life-years for 29 cancer groups, 1990 to 2016: a systematic analysis for the global burden of disease study. JAMA Oncol. 2018:4:1553-68.

\section{Publisher's Note}

Springer Nature remains neutral with regard to jurisdictional claims in published maps and institutional affiliations.

Ready to submit your research? Choose BMC and benefit from:
- fast, convenient online submission
- thorough peer review by experienced researchers in your field
- rapid publication on acceptance
- support for research data, including large and complex data types
- gold Open Access which fosters wider collaboration and increased citations
- maximum visibility for your research: over 100M website views per year
At BMC, research is always in progress.
Learn more biomedcentral.com/submissions

